\title{
Association between pre- and perinatal exposures and Tourette syndrome or chronic tic disorder in the ALSPAC cohort ${ }^{\dagger}$
}

Carol A. Mathews, Jeremiah M. Scharf, Laura L. Miller, Corrie Macdonald-Wallis, Debbie A. Lawlor and Yoav Ben-Shlomo

\section{Background}

Tourette syndrome and chronic tic disorder are heritable but aetiologically complex. Although environment plays a role in their development, existing studies of non-genetic risk factors are inconsistent.

\section{Aims \\ To examine the association between pre- and perinatal exposures and Tourette syndrome/chronic tic disorder in the Avon Longitudinal Study of Parents and Children (ALSPAC) prospective longitudinal pre-birth cohort.}

\section{Method}

Relationships between exposures and Tourette syndrome/ chronic tic disorder were examined in 6090 children using logistic regression.

\section{Results}

Maternal alcohol and cannabis use, inadequate maternal weight gain and parity were associated with Tourette syndrome or Tourette syndrome/chronic tic disorder. Other previously reported exposures, including birth weight and prenatal maternal smoking, were not associated with Tourette syndrome/chronic tic disorder.

\section{Conclusions}

This study supports previously reported relationships between Tourette syndrome/chronic tic disorder and prenatal alcohol exposure, and identifies additional previously unexplored potential prenatal risk factors.

\section{Declaration of interest}

C.A.M. and J.M.S. have received travel support from the Tourette Syndrome Association (TSA). C.A.M. is a member of the TSA Medical Advisory Board.
Tourette syndrome is a phenomenologically heterogeneous neuropsychiatric disorder comprised of multiple motor and vocal tics that begin in early childhood and persist for at least a year. ${ }^{1}$ Chronic tic disorder is a highly related condition, with similar clinical and demographic characteristics, consisting of either only chronic motor or only vocal tics, but not both. ${ }^{1}$ Tourette syndrome has a population prevalence of $0.3-0.8 \%$, whereas chronic tic disorder has a population prevalence of $1-3 \%$ (summarised in Scharf et al). ${ }^{2}$ Although varying widely in clinical presentation and impact, these disorders can cause significant physical and psychosocial morbidity, particularly in childhood, and in severe cases produce lifelong disability. ${ }^{3}$ Tourette syndrome and chronic tic disorder have a strong genetic component, and together these disorders have among the highest familial recurrence risks of all neurodevelopmental disorders. ${ }^{4}$ However, despite the high heritabilities, twin and family studies have consistently demonstrated a significant role for additional, nongenetic factors in the pathogenesis of Tourette syndrome and chronic tic disorder. ${ }^{4-9}$

Although a number of studies have examined the potential contributions of various non-genetic factors in the development of Tourette syndrome, the results have been conflicting, and the potential biological mechanisms for the identified risk factors are unclear. ${ }^{7,10-14}$ Maternal prenatal smoking, complications during pregnancy, birth weight, gestational age, Apgar scores at 5 min after birth, number of prenatal visits, younger maternal age and older paternal age have all been reported to be associated with risk of Tourette syndrome. ${ }^{12,14-17}$ Low birth weight, maternal nausea and vomiting in the first trimester, maternal life stress and maternal smoking during pregnancy have also been associated with increased tic severity. ${ }^{5,10,11}$ Lastly, increased rates of co-occurring

†See editorial, pp. 6-8, this issue. obsessive-compulsive disorder (OCD) or attention-deficit hyperactivity disorder (ADHD) in Tourette syndrome have been reported to be associated with low birth weight, forceps delivery and either coffee, alcohol or tobacco use during pregnancy. ${ }^{10,13}$ Unfortunately, for the most part, the environmental risk factors identified have not been replicated, although some association between prenatal maternal smoking and Tourette syndrome, tic severity or comorbid conditions such as OCD or ADHD has been identified in multiple studies. ${ }^{10,13,14,18,19}$

Although providing hints about potential environmental contributors to chronic tic disorder, most of the previously published studies have methodological limitations, including retrospective study designs, ${ }^{5,10-13,16-18,20}$ the absence of a control group of individuals without Tourette syndrome/chronic tic disorder, ${ }^{5,10-13}$ limited number of variables examined ${ }^{5,10-18,20}$ and/or ascertainment of cases from specialty clinics rather than from the general population. ${ }^{5,10-14,16,18,20}$ The aim of this work, the largest prospective study to date, was to (a) attempt to replicate the previous observational associations between tic disorders and non-genetic risk factors, and (b) examine a number of additional, previously unexplored, potential risk factors for tic disorders using the Avon Longitudinal Study of Parents and Children (ALSPAC). We hypothesised, based on the previous literature, that maternal prenatal smoking and alcohol use, excessive vomiting during pregnancy, complications of delivery (e.g. assisted delivery) and low birth weight or young gestational age at birth would be associated with Tourette syndrome and chronic tics in this population.

\section{Method}

\section{Participants}

The ALSPAC is an ongoing, prospective, population-based, prebirth cohort study of all children born to 14541 pregnant women 
resident in Avon, UK (representing 85\% of the eligible population), with expected dates of delivery between 1 April 1991 and 31 December $1992 .^{21,22}$ Ethical approval for the study was obtained from the ALSPAC Law and Ethics Committee and the local research ethics committees. The characteristics of the population-based ALSPAC sample and its generalisability have been previously reported ${ }^{21,22}$ and are summarised in the online supplement.

\section{Outcome measures}

Data were collected prospectively through maternal questionnaires, supplemented by obstetric and paediatric medical records that began at the time of enrolment and continued throughout pregnancy and at least yearly thereafter (see the online supplement). The primary outcome measures were diagnoses of Tourette syndrome or chronic tic disorder at age 13 or 14 , based on DSM-IV-TR criteria ${ }^{1}$ and were derived from the tic-related questions in the maternal questionnaires (summarised in Scharf et al). ${ }^{2}$ Children with intellectual disability or autism were excluded from the sample. The final sample for this study consisted of 6090 children, with data available at ages 13-14 including 50 with Tourette syndrome, 72 with chronic tic disorder (for a total of 122 with either Tourette syndrome or chronic tic disorder) and 5968 with no tics (online Fig. DS1). To compensate for the relatively small sample sizes, two nested phenotypes were examined: Tourette syndrome and either Tourette syndrome or chronic tic disorder (Tourette syndrome/chronic tic disorder). Power calculations for representative predictor variables of interest are provided in the online supplement.

\section{Predictor variables/exposures}

Exposures of interest included prenatal and perinatal factors previously reported to be associated with either Tourette syndrome or tic severity in at least one previous study (confirmatory analyses), including parental age, maternal use of tobacco, alcohol or caffeine, and excessive nausea or vomiting during pregnancy. We also examined factors thought to be relevant to other related neurodevelopmental disorders such as ADHD (exploratory analyses), for example, gestational diabetes, maternal weight gain during pregnancy, and parity. For the confirmatory analyses, we examined the previously reported exposure (e.g. maternal nausea and vomiting) and the available related exposures (e.g. medication use for nausea/vomiting). Exposures were divided into five thematic categories: pre-existing parental fitness; early pregnancy exposures; toxic exposures; complications of pregnancy; and complications of delivery (see the online supplement for definitions of predictor variables). Pre-existing parental fitness included maternal and paternal age at child's birth, maternal baseline pre-pregnancy weight, preexisting diabetes, maternal smoking before pregnancy, cannabis or alcohol use before pregnancy, and parity. Early pregnancy exposures included prior miscarriage or stillborn birth, hyperemesis of pregnancy, vomiting in the first trimester, and medications for vomiting in the first trimester. Toxic exposures were examined in the first, second and third trimesters separately, and included maternal influenza infection, substance use during pregnancy (nicotine, cannabis, alcohol, caffeine and illicit drugs) and partner smoking during and at the beginning of pregnancy. Complications of pregnancy included diabetes in pregnancy, ${ }^{23}$ hypertensive disorder of pregnancy, ${ }^{24,25}$ vomiting or medications for vomiting in the second or third trimesters, placental weight (adjusted for birth weight), total maternal weight gain (both observed and predicted $)^{26}$ and twin or multiple births. Complications of delivery included method of delivery, Apgar scores of $<7$ at
$5 \mathrm{~min}$, inspired oxygen or mechanical ventilation at birth, resuscitation, pre-term labour or premature rupture of membranes, gestation of $<37$ or $\geqslant 42$ weeks, and birth weight (in grams) were adjusted for gestational age. Maternal and paternal education and maternal marital status (ever married, and married at time of child's birth) were examined as potential confounders; as they were not significantly associated with Tourette syndrome/chronic tic disorder, they were not included as covariates in the analyses.

\section{Analyses}

Analyses were conducted in a step-wise process, using $P<0.10$ for Tourette syndrome or Tourette syndrome/chronic tic disorder as the criterion for progression to the next step (online Fig. DS2). Variables with opposite direction of effects between Tourette syndrome and Tourette syndrome/chronic tic disorder were not retained for further analyses. The exception was maternal age, which was included in relevant multivariable models as a confounder. Univariable analyses were conducted using logistic regression, and any previously identified (confirmatory) predictor variables associated with Tourette syndrome or Tourette syndrome/chronic tic disorder at $P<0.05$ were noted. Exploratory risk factors were only examined for association with Tourette syndrome or Tourette syndrome/chronic tic disorder after appropriate multivariable adjustment. In stage 2 , all confirmatory or exploratory predictor variables that met the threshold criteria of $P<0.10$ in univariable analysis were subjected to logistic regression including socioeconomic status (SES) as a covariate, as we have previously shown that low SES is associated with increased risk of Tourette syndrome/chronic tic disorder. ${ }^{27}$ Additional covariates were also included as potential confounders (e.g. given the relationship between maternal age and substance use, maternal age was included in analyses of smoking, drug, or alcohol use).

As exposures within each thematic category might be related mechanistically (e.g. maternal vomiting may cause low weight gain during pregnancy), we examined the independent effects of each variable with $P<0.10$ in the stage 2 analyses via multivariable regressions within their respective categories (e.g. maternal fitness, complications of pregnancy). For two co-linear variables, only the most strongly associated was included in the multivariable analyses. Finally, any variables or confounders that showed a trend for association $(P<0.10)$ in the category-specific multivariable models were examined in a final global model. Variables with $P<0.05$ for either Tourette syndrome or Tourette syndrome/ chronic tic disorder in the global model were interpreted as putative risk factors, with highest confidence placed in confirmatory variables, as well as exploratory variables with the same direction of effect for both nested phenotypes.

\section{Results}

\section{Confirmatory analyses}

Of the variables previously associated with increased Tourette syndrome/chronic tic disorder risk or severity, maternal age, paternal age, assisted delivery, maternal alcohol use in the last 2 months of pregnancy and a combined variable examining any maternal smoking, caffeine or alcohol use in the third trimester were associated with Tourette syndrome and/or Tourette syndrome/chronic tic disorder at $P<0.05$ in unadjusted univariable analyses in the ALSPAC sample (see online Table DS1). Younger maternal and paternal ages were associated with increased odds of Tourette syndrome/chronic tic disorder ( $P=0.003$ and $P=0.02$ respectively), but not with Tourette syndrome alone. Greater than two units of alcohol use per week 
in the last 2 months of pregnancy was associated with an increased risk of Tourette syndrome alone $(P=0.03)$ but not Tourette syndrome/chronic tic disorder, and any use of alcohol, tobacco or caffeine in the third trimester was associated with an increased risk of both Tourette syndrome and Tourette syndrome/chronic tic disorder ( $P=0.05$ and $P=0.01$ respectively). Assisted delivery was associated with an increased risk of Tourette syndrome $(P=0.05)$ but not Tourette syndrome/chronic tic disorder. No other previously reported risk factors were associated with Tourette syndrome/chronic tic disorder in the ALSPAC sample. Following adjustment for SES, only paternal age, maternal alcohol use in the last 2 months of pregnancy and maternal smoking, alcohol or caffeine use in the third trimester remained nominally associated with either Tourette syndrome or Tourette syndrome/ chronic tic disorder (online Tables DS1 and DS2). In addition, although most associations decreased in strength after adjusting for SES, the association for medications for vomiting in the second trimester increased slightly (unadjusted odds ratio $(\mathrm{OR})=2.2 ; P=0.07$; SES-adjusted $\mathrm{OR}=2.31 ; P=0.05)$.

\section{Exploratory risk factors}

Four of the exploratory risk factors were nominally associated with either Tourette syndrome or Tourette syndrome/chronic tic disorder in the ALSPAC sample (Table 1). These risk factors included parity (for Tourette syndrome/chronic tic disorder), maternal cannabis use during pregnancy, particularly the last
2 months of pregnancy, for both Tourette syndrome and Tourette syndrome/chronic tic disorder, any illegal substance use in pregnancy (for Tourette syndrome) and less than adequate predicted weight gain during pregnancy. None of the other exploratory variables were associated with Tourette syndrome or with Tourette syndrome/chronic tic disorder.

\section{Multivariable analyses}

Individual variables with $P<0.10$ in SES-adjusted univariable analyses were examined jointly in multivariable models grouped into maternal fitness, complications of pregnancy and toxic exposures categories. Paternal age and maternal age were found to be co-linear, and therefore paternal age was excluded from subsequent models. Similarly, assisted delivery was not retained because the direction of association between this variable and Tourette syndrome and Tourette syndrome/chronic tic disorder were not consistent, suggesting that the identified trend for association was due to stochastic variation.

In the parental fitness category, only parity remained independently associated with Tourette syndrome/chronic tic disorder, with later births associated with lower risk (Table 2). For complications of pregnancy, less than adequate weight gain during pregnancy remained independently associated with Tourette syndrome and Tourette syndrome/chronic tic disorder. For toxic exposures, maternal alcohol and cannabis use in the last 2 months of pregnancy remained independently associated with

\begin{tabular}{|c|c|c|c|c|c|c|c|c|}
\hline \multirow[b]{3}{*}{ Exposure } & \multicolumn{4}{|c|}{ Tourette syndrome only } & \multicolumn{4}{|c|}{ Tourette syndrome/chronic tic disorder } \\
\hline & \multicolumn{2}{|l|}{ Unadjusted } & \multicolumn{2}{|c|}{ Adjusted for SES } & \multicolumn{2}{|l|}{ Unadjusted } & \multicolumn{2}{|c|}{ Adjusted for SES } \\
\hline & OR $(95 \% \mathrm{Cl})$ & $P$ & OR $(95 \% \mathrm{Cl})$ & $P$ & OR $(95 \% \mathrm{Cl})$ & $P$ & OR $(95 \% \mathrm{Cl})$ & $P$ \\
\hline \multicolumn{9}{|l|}{ Maternal fitness } \\
\hline Pre-pregnancy weight, kg & $1.01(0.98-1.04)$ & 0.43 & & & $1.01(0.99-1.02)$ & 0.41 & & \\
\hline Existing diabetes & $0.37(0.05-2.78)$ & 0.34 & & & $0.46(0.11-1.91)$ & 0.29 & & \\
\hline Maternal smoking before pregnancy & $1.28(0.68-2.38)$ & 0.45 & $1.33(0.69-2.57)$ & 0.40 & $1.43(0.97-2.13)$ & 0.07 & $1.30(0.85-1.99)$ & 0.22 \\
\hline Cannabis use before pregnancy & $2.43(0.86-6.84)$ & 0.09 & $2.38(0.83-6.81)$ & 0.11 & $1.46(0.64-3.73)$ & 0.37 & $1.32(0.56-3.08)$ & 0.52 \\
\hline Parity (second or later born $v$. first born) & & & $0.60(0.33-1.11)$ & 0.10 & & & $0.62(0.42-0.92)$ & 0.02 \\
\hline \multicolumn{9}{|l|}{$\begin{array}{l}\text { Early pregnancy exposures, prior } \\
\text { miscarriage/stillborn }\end{array}$} \\
\hline \multirow{2}{*}{\multicolumn{9}{|c|}{$\begin{array}{l}\text { Toxic exposures } \\
\text { Influenza }\end{array}$}} \\
\hline & & & & & & & & \\
\hline 1st trimester & $1.01(0.36-2.82)$ & 0.99 & & & $0.86(0.41-1.77)$ & 0.68 & & \\
\hline 2nd trimester & $0.81(0.19-3.35)$ & 0.77 & & & $1.38(0.67-2.86)$ & 0.39 & & \\
\hline 3rd trimester & $1.49(0.46-4.84)$ & 0.50 & & & $1.23(0.54-2.84)$ & 0.62 & & \\
\hline Anytime in pregnancy & $1.12(0.52-2.39)$ & 0.78 & & & $1.23(0.76-1.97)$ & 0.40 & & \\
\hline Cannabis use in 1st trimester & $4.74(1.67-13.44)$ & 0.003 & $4.68(1.61-13.63)$ & 0.005 & $1.87(0.68-5.17)$ & 0.23 & $1.68(0.60-4.71)$ & 0.32 \\
\hline Cannabis use in last 2 months of pregnancy & $6.37(2.23-18.21)$ & 0.001 & $6.00(2.06-17.50)$ & 0.001 & $3.11(1.23-7.84)$ & 0.016 & $2.82(1.10-7.22)$ & 0.03 \\
\hline Any illegal drug use in pregnancy & $4.28(1.51-12.11)$ & 0.006 & $4.20(1.45-12.16)$ & 0.01 & $2.10(0.84-5.25)$ & 0.11 & $1.93(0.76-4.87)$ & 0.17 \\
\hline Partner smoked at start of pregnancy & $1.50(0.77-2.95)$ & 0.24 & & & $1.24(0.80-1.93)$ & 0.33 & & \\
\hline \multicolumn{9}{|l|}{ Pregnancy complications } \\
\hline Diabetes in pregnancy & $1.66(0.51-5.38)$ & 0.40 & & & $1.14(0.46-2.81)$ & 0.78 & & \\
\hline Hypertensive disorder of pregnancy & $0.98(0.46-2.10)$ & 0.96 & & & $1.01(0.62-1.68)$ & 0.95 & & \\
\hline Placental weight (adjusted by birth weight), $g$ & $1.00(1.00-1.00)$ & 0.57 & & & $1.00(0.99-1.00)$ & 0.96 & & \\
\hline Twin $v$. singleton & $0.99(0.14-7.26)$ & 0.99 & & & $1.23(0.39-3.92)$ & 0.73 & & \\
\hline \multicolumn{9}{|l|}{ Predicted weight gain ${ }^{b}$} \\
\hline Less than adequate & $5.69(1.99-16.25)$ & 0.001 & $9.70(2.71-34.73)$ & $<0.0001$ & $2.37(1.22-4.60)$ & 0.01 & $3.18(1.52-6.65)$ & 0.002 \\
\hline More than adequate & $1.98(0.74-5.30)$ & 0.17 & $2.95(0.88-10.05)$ & 0.09 & $1.27(0.73-2.20)$ & 0.40 & $1.54(0.82-2.89)$ & 0.18 \\
\hline \multicolumn{9}{|l|}{ Complications of delivery } \\
\hline Inspired oxygen & NA & NA & & & $0.66(0.09-4.82)$ & 0.69 & & \\
\hline Mechanical ventilation & NA & NA & & & $1.35(0.18-9.98)$ & 0.77 & & \\
\hline Resuscitation & $0.28(0.04-2.01)$ & 0.21 & & & $1.15(0.58-2.30)$ & 0.69 & & \\
\hline Preterm labour/PROM & NA & NA & & & $1.64(0.22-12.09)$ & 0.63 & & \\
\hline
\end{tabular}


Table 2 Multivariable associations examining maternal fitness, pregnancy complications and potentially toxic exposures and

Tourette syndrome/chronic tic disorder risk

\begin{tabular}{|c|c|c|c|c|}
\hline & \multicolumn{2}{|c|}{ Tourette syndrome } & \multicolumn{2}{|c|}{ Tourette syndrome/chronic tic disorder } \\
\hline & $\mathrm{OR}(95 \% \mathrm{Cl})$ & $P$ & OR $(95 \% \mathrm{Cl})$ & $P$ \\
\hline \multicolumn{5}{|l|}{ Maternal fitness ${ }^{a}$} \\
\hline Maternal age $\mathrm{e}^{\mathrm{b}}$ & $2.83(0.81-9.95)$ & 0.10 & $1.35(0.47-3.86)$ & 0.58 \\
\hline Parity ${ }^{c}$ & $0.65(0.41-1.03)$ & 0.07 & $0.72(0.54-0.95)$ & 0.02 \\
\hline \multicolumn{5}{|l|}{ Pregnancy complications $^{\mathrm{d}}$} \\
\hline Less than adequate weight gain & $4.55(1.67-12.41)$ & $<0.01$ & $2.05(1.05-4.00)$ & 0.04 \\
\hline More than adequate weight gain & $1.80(0.72-4.47)$ & 0.21 & $1.35(0.81-2.29)$ & 0.25 \\
\hline Medications for vomiting in 2nd trimester & $1.13(0.15-8.41)$ & 0.91 & $2.13(0.76-5.96)$ & 0.15 \\
\hline \multicolumn{5}{|l|}{ Toxic exposures $^{d}$} \\
\hline Smoking in last 2 months of pregnancy & $0.93(0.48-1.80)$ & 0.82 & $1.20(0.82-1.77)$ & 0.34 \\
\hline Alcohol use in last 2 months of pregnancy & $1.57(1.05-2.33)$ & 0.03 & $1.24(0.94-1.64)$ & 0.12 \\
\hline Cannabis use in last 2 months of pregnancy & $4.60(1.28-16.62)$ & 0.02 & $1.91(0.56-6.49)$ & 0.30 \\
\hline \multicolumn{5}{|c|}{$\begin{array}{l}\text { a. Mutually adjusted for other variables in the model and socioeconomic status level. } \\
\text { b. Maternal age odds ratios (ORs) are denoted as change per } 10 \text { years of mother's age. } \\
\text { c. Parity is defined as second born or later compared with first born as the reference category. } \\
\text { d. Mutually adjusted for other variables in the model, maternal age and socioeconomic status level. }\end{array}$} \\
\hline
\end{tabular}

Tourette syndrome, but not with Tourette syndrome/chronic tic disorder. Maternal prenatal smoking, although not associated with Tourette syndrome or Tourette syndrome/chronic tic disorder in this sample, was included in the multivariable model because of the frequent association between smoking and alcohol use. ${ }^{28}$

The final global model incorporated all of the pre- and perinatal variables that continued to show an association with Tourette syndrome or Tourette syndrome/chronic tic disorder in the multivariable analyses described above (Table 3). In this final model, which also included SES, maternal age and maternal smoking during pregnancy as confounders, only weight gain during pregnancy was consistently associated with both Tourette syndrome and Tourette syndrome/chronic tic disorder, with threeto sixfold increases in the odds of Tourette syndrome/chronic tic disorder or Tourette syndrome for less than adequate weight gain ( $P=0.006$ for Tourette syndrome and $P=0.005$ for Tourette syndrome/chronic tic disorder). More than adequate weight gain also continued to show a trend for association with both outcome measures, with two- to threefold increases in risk for Tourette syndrome/chronic tic disorder and Tourette syndrome $(P=0.05$ and $P=0.08$ respectively). Similarly, alcohol and cannabis use in the last 2 months of pregnancy showed associations with Tourette syndrome $(\mathrm{OR}=1.72, P=0.01$ and $\mathrm{OR}=4.30, P=0.03$ respectively) and somewhat weaker associations with Tourette syndrome/chronic tic disorder in the same direction, whereas parity showed consistent association with both Tourette syndrome and Tourette syndrome/ chronic tic disorder, although only the Tourette syndrome/chronic tic disorder association remained nominally significant in the final model $(\mathrm{OR}=0.57, P=0.07$ for Tourette syndrome; $\mathrm{OR}=0.66$, $P=0.03$ for Tourette syndrome/chronic tic disorder).

\section{Discussion}

To our knowledge, this is the first comprehensive, large-scale, prospective study examining potential environmental risk factors for chronic tic disorder. Using an association threshold at $P<0.05$ for either Tourette syndrome or Tourette syndrome/ chronic tic disorder in the global multivariable model and the same direction of effect for both nested phenotypes as our metric, the results of this study support an association between Tourette syndrome/chronic tic disorder and only one of the previously reported prenatal risk factors, alcohol use during pregnancy. ${ }^{10,15,17}$ We found no associations between Tourette syndrome/chronic tic disorder and the other prior candidate risk factors, most importantly, prenatal maternal smoking, low birth weight, gestational age and complications of delivery. ${ }^{5,7,10-13}$ We also found no consistent effect of parental age. Both younger maternal and paternal age were associated with Tourette syndrome/chronic tic disorder in the univariable analyses, but the direction of association changed when related variables (parity, maternal age and paternal age) were included in the multivariable analyses. This result, and the fact that parental age difference was not associated with Tourette syndrome/chronic tic disorder, suggests that the previous associations most likely reflect confounding and chance.

Of note, in our initial confirmatory analyses, five previously reported prenatal risk factors (maternal age, paternal age, assisted

Table 3 Multivariable associations examining all variables associated with Tourette syndrome or Tourette syndrome/chronic tic disorder in the parental fitness, complications of pregnancy and toxic exposures analyses ${ }^{a}$

\begin{tabular}{|c|c|c|c|c|}
\hline & \multicolumn{2}{|c|}{ Tourette syndrome } & \multicolumn{2}{|c|}{ Tourette syndrome/chronic tics } \\
\hline & Odds ratio $(95 \% \mathrm{Cl})$ & $P$ & Odds ratio $(95 \% \mathrm{Cl})$ & $P$ \\
\hline Maternal age & $4.31(0.86-21.67)$ & 0.08 & $1.42(0.31-6.43)$ & 0.65 \\
\hline Parity & $0.57(0.31-1.05)$ & 0.07 & $0.66(0.45-0.97)$ & 0.03 \\
\hline Less than adequate weight gain & $6.66(1.74-25.51)$ & $<0.01$ & $3.30(1.43-7.65)$ & $<0.01$ \\
\hline More than adequate weight gain & $3.03(0.88-10.39)$ & 0.08 & $2.03(1.00-4.11)$ & 0.05 \\
\hline Alcohol use in last 2 months of pregnancy & $1.72(1.13-2.62)$ & 0.01 & $1.27(0.95-1.69)$ & 0.11 \\
\hline Smoking in last 2 months of pregnancy & $0.99(0.51-1.92)$ & 0.97 & $1.25(0.82-1.89)$ & 0.30 \\
\hline Cannabis use last 2 months of pregnancy & $4.30(1.14-16.22)$ & 0.03 & $2.01(0.57-7.01)$ & 0.28 \\
\hline
\end{tabular}


delivery, maternal alcohol use, and maternal smoking, caffeine or alcohol use in the second trimester) were nominally associated with either Tourette syndrome or Tourette syndrome/chronic tic disorder in unadjusted univariable analyses. However, after adjustment for SES and other appropriate confounders, only maternal alcohol use in the last 2 months of pregnancy and maternal smoking, caffeine or alcohol use in the last trimester remained associated with Tourette syndrome or Tourette syndrome/chronic tic disorder. This finding suggests that the previously reported risk factors may have been confounded by unmeasured or incompletely measured variables, including SES. This highlights a major strength of the ALSPAC cohort, specifically the availability of extensive, detailed, prospectively collected, individual-level data to limit confounding.

We also identified three new potential prenatal risk factors for chronic tic disorders that to our knowledge have not been previously examined: cannabis use during pregnancy (independent of maternal alcohol and tobacco use), parity and inadequate weight gain during pregnancy (independent of the child's birth weight or gestational status). Of these, the strongest association was for inadequate weight gain during pregnancy, which was associated both with Tourette syndrome and with Tourette syndrome/chronic tic disorder in the final model.

In contrast to previous hypotheses (e.g. Mathews et al), ${ }^{10}$ the risk factors identified here do not fit easily into one mechanistic model (e.g. chronic hypoxia leading to basal ganglia damage in utero). Our findings, which remain strong even when variables from all three categories are simultaneously examined, suggest that there may be several types of environmental exposures that can interact with genetic susceptibility factors to increase the risk of chronic tic disorder. The mechanisms underlying these associations are not known, and some of the patterns observed in our study are not easily explained. For example, the associations between Tourette syndrome/chronic tic disorder, inadequate maternal weight gain and parity (being the first born child) ${ }^{29}$ may suggest a less-than-optimal intra-uterine environment for the developing fetus, although the lack of association between Tourette syndrome/chronic tic disorder, birth weight and younger gestational age at birth stand somewhat in contradiction to this hypothesis.

The associations between prenatal maternal cannabis and alcohol use appear to be more straightforward, and may represent direct toxic effects on the developing fetal brain, such as is seen in fetal alcohol syndrome. ${ }^{30}$ Although phenotypically distinct from Tourette syndrome, both fetal alcohol syndrome and Tourette syndrome are neurodevelopmental disorders that manifest in early childhood, and what is understood of the mechanisms behind the development of fetal alcohol syndrome ${ }^{30}$ may serve as a starting point for the investigation of how toxic exposures such as alcohol and cannabis might lead to the development of chronic tic disorder as well.

Several of the risk factors identified here have also been suggested as risk factors for other neurodevelopmental disorders such as autism and ADHD. ${ }^{15,31,32}$ This is not surprising, given the high degree of phenotypic overlap between these disorders and the emerging evidence for shared aetiologies across neurodevelopmental disorders. ${ }^{33}$ The aetiology of tic disorders and of other neurodevelopmental disorders is clearly complex, with both shared and unique environmental and genetic factors contributing to their development.

\section{Limitations}

The primary limitation of this study relates to sample size. Although it is the largest study reported to date examining potential environmental risk factors for chronic tic disorders, the number of children identified in the ALSPAC cohort with
Tourette syndrome or chronic tic disorder was fairly low. This is likely due to the low prevalence of these disorders in the general population and is an inherent limitation of population-based studies. The small number of cases leads to a relative lack of power, which may prevent the identification of true Tourette syndrome/chronic tic disorder risk factors that are uncommon in the study population (e.g. twin or premature births). We have compensated for this to the extent possible by examining two nested phenotypes, Tourette syndrome and Tourette syndrome/ chronic tic disorder, and focusing on variables that showed the same direction of effect for both. This relative lack of power is counterbalanced by the many other advantages of this population-based cohort, most notably the prospective data collection, the comprehensiveness of the environmental risk variables available for study, and the depth, complexity and precision of the data (e.g. the use of multiple informants as well as the availability of medical and education records). This advantage has allowed us to definitively exclude proposed risk factors such as low birth weight from having a moderate or large effect on Tourette syndrome/chronic tic disorder risk, at least in the ALSPAC sample, as this was precisely determined from obstetric records for all children in the study.

The small sample size also does not allow us to differentiate between false positives and differential association between Tourette syndrome (the more extreme phenotype) and Tourette syndrome/chronic tic disorder (less extreme). Some of the putative risk factors in the global model had stronger associations with Tourette syndrome than with Tourette syndrome/chronic tic disorder (e.g. maternal alcohol and cannabis use), whereas for others (e.g. parity), the effect was stronger for Tourette syndrome/chronic tic disorder than for Tourette syndrome. However, the directions of effect for Tourette syndrome and Tourette syndrome/chronic tic disorder were consistent for all of these variables, suggesting that the associations are less likely to be due to chance. Similarly, we cannot exclude the possibility that our associations may reflect residual confounding despite our best attempts to adjust for potential confounders. This is an inevitable limitation of observational analyses.

\section{Implications}

This study suggests several potentially modifiable environmental risk factors for Tourette syndrome and chronic tic disorder. One previously reported risk factor, alcohol use in the third trimester of pregnancy, remained significant in the final adjusted model and could thus be considered a replicated prenatal risk factor for Tourette syndrome and chronic tic disorder. Others are novel and require replication in other cohorts. As parallel efforts to identify Tourette syndrome susceptibility genes in large cohorts are also underway, a logical next step would be to examine the interaction between environmental factors such as prenatal maternal alcohol use and future definitive Tourette syndrome genetic susceptibility alleles. With the extensive environmental and phenotypic data, along with genetic data, the ALSPAC cohort provides an excellent opportunity for examinations of gene $\times$ environment interactions such as these in the future.

\section{Funding}

This research was funded by grants from the Tourette Syndrome Association, National Institute for Neurological Disorders and Stroke (R01 NS444653 and U01 NS40024), National Institute for Mental Health (K23 MH085057), United States National Institutes of Health (NIH) National Institute of Diabetes and Digestive and Kidney Diseases (R01 DK077659) and the National Institute of Diabetes and Digestive and Kidney Diseases (R01 DK077659) and the
Wellcome Trust (WT087997MA). The latter also funded the salary of C.M-W. in relation to her work on this paper. 


\section{Acknowledgements}

The UK Medical Research Council and the Wellcome Trust (Grant ref: 092731) and the University of Bristol provide core support for ALSPAC. This publication is the work of the authors and they will serve as guarantors for the contents of this paper. We are extremely grateful to all the families who took part in this study, the midwives for their help in recruiting them and the whole ALSPAC team, which includes interviewers, computer and laboraing them and the whole ALSPAC team, which includes interviewers, computer and labora-
tory technicians, clerical workers, research scientists, volunteers, managers, receptionists tory technicians,

Carol A. Mathews, MD, Program for Genetics and Epidemiology of Neuropsychiatric Symptoms, Department of Psychiatry, University of California San Francisco, San Francisco, California, USA; Jeremiah M. Scharf, MD, PhD, Psychiatric and Neurodevelopmental Genetics Unit, Center for Human Genetics Research, Massachusetts General Hospital, Boston, Massachusetts; Departments of Psychiatry and Neurology, Massachusetts General Hospital, Boston, Massachusetts; Division of Cognitive and Behavioral Neurology, Department of Neurology, Brigham and Women's Hospital, Boston, Massachusetts, USA; Laura L. Miller, MSC, and Women's Hospital, Boston, Massachusetts, USA; Laura L. Miller, MSC,
school of Social and Community Medicine, University of Bristol, UK; Corrie Macdonald-Wallis, PhD, Debbie A. Lawlor, PhD, School of Social and Community Medicine, and MRC Centre for Causal Analyses in Translational Epidemiology, University of Bristol, UK: Yoav Ben-Shlomo, MD, PhD, School of Social and Community Medicine, University of Bristol, UK

Correspondence: Carol A. Mathews, MD, Department of Psychiatry, University of California, San Francisco, 401 Parnassus Ave, San Francisco, CA 94143-0984, USA. Email: cmathews@Ippi.ucsf.edu

First received 20 Dec 2012, final revision 9 May 2013, accepted 18 Jul 2013

\section{References}

1 American Psychiatric Association. Diagnostic and Statistical Manual of Mental Disorders (4th edn, Text Revision) (DSM-IV-TR). APA, 2000.

2 Scharf JM, Miller LL, Mathews CA, Ben-Shlomo Y. Prevalence of tourette syndrome and chronic tics in the population-based avon longitudinal study of parents and children cohort. J Am Acad Child Adolesc Psychiatry 2012; 51 192-201.

3 Elstner K, Selai CE, Trimble MR, Robertson MM. Quality of Life (QOL) of patients with Gilles de la Tourette's syndrome. Acta Psychiatr Scand 2001; 103: 52-9.

4 O'Rourke JA, Scharf JM, Yu D, Pauls DL. The genetics of Tourette syndrome: a review. J Psychosom Res 2009; 67: 533-45.

5 Hyde TM, Aaronson BA, Randolph C, Rickler KC, Weinberger DR. Relationship of birth weight to the phenotypic expression of Gilles de la Tourette's syndrome in monozygotic twins. Neurology 1992; 42 (3 Pt 1): 652-8.

6 Price RA, Kidd KK, Cohen DJ, Pauls DL, Leckman JF. A twin study of Tourette syndrome. Arch Gen Psychiatry 1985; 42: 815-20.

7 Walkup JT. Epigenetic and environmental risk factors in Tourette syndrome. Adv Neurol 2001; 85: 273-9.

8 Seuchter SA, Hebebrand J, Klug B, Knapp M, Lehmkuhl G, Poustka F, et al. Complex segregation analysis of families ascertained through Gilles de la Tourette syndrome. Genet Epidemiol 2000; 18: 33-47.

9 Walkup JT, LaBuda MC, Singer HS, Brown J, Riddle MA, Hurko O. Family study and segregation analysis of Tourette syndrome: evidence for a mixed model of inheritance. Am J Hum Genet 1996; 59: 684-93.

10 Mathews CA, Bimson B, Lowe TL, Herrera LD, Budman CL, Erenberg G, et al. Association between maternal smoking and increased symptom severity in Tourette's syndrome. Am J Psychiatry 2006; 163: 1066-73.

11 Leckman JF, Dolnansky ES, Hardin MT, Clubb M, Walkup JT, Stevenson J, et al. Perinatal factors in the expression of Tourette's syndrome: an exploratory study. J Am Acad Child Adolesc Psychiatry 1990; 29: 220-6.

12 Leckman JF, Price RA, Walkup JT, Ort S, Pauls DL, Cohen DJ. Nongenetic factors in Gilles de la Tourette's syndrome. Arch Gen Psychiatry 1987; 44 100.

13 Santangelo SL, Pauls DL, Goldstein JM, Faraone SV, Tsuang MT, Leckman JF. Tourette's syndrome: what are the influences of gender and comorbid obsessive-compulsive disorder? J Am Acad Child Adolesc Psychiatry 1994; 33: $795-804$.
14 Whitaker AH, Van Rossem R, Feldman JF, schonfeld IS, Pinto-Martin JA, Tore $\mathrm{C}$, et al. Psychiatric outcomes in low-birth-weight children at age 6 years: relation to neonatal cranial ultrasound abnormalities. Arch Gen Psychiatry 1997; 54: 847-56.

15 Klug MG, Burd L, Kerbeshian J, Benz B, Martsolf JT. A comparison of the effects of parental risk markers on pre- and perinatal variables in multiple patient cohorts with fetal alcohol syndrome, autism, Tourette syndrome, and sudden infant death syndrome: an enviromic analysis. Neurotoxicol Teratol 2003; 25: 707-17.

16 Pasamanick B, Kawi A. A study of the association of prenatal and paranatal factors with the development of tics in children; a preliminary investigation. J Pediatr 1956; 48: 596-601.

17 Burd L, Severud R, Klug MG, Kerbeshian J. Prenatal and perinatal risk factors for Tourette disorder. J Perinat Med 1999; 27: 295-302.

18 Motlagh MG, Katsovich L, Thompson N, Lin H, Kim YS, Scahill L, et al. Severe psychosocial stress and heavy cigarette smoking during pregnancy: an examination of the pre- and perinatal risk factors associated with ADHD and Tourette syndrome. Eur Child Adolesc Psychiatry 2010; 19: 755-64.

19 Pringsheim $\mathrm{T}$, Sandor $\mathrm{P}$, Lang $\mathrm{A}$, Shah $\mathrm{P}, \mathrm{O}$ 'Connor P. Prenatal and perinatal morbidity in children with Tourette syndrome and attention-deficit hyperactivity disorder. J Dev Behav Pediatr 2009; 30: 115-21.

20 Bos-Veneman NG, Kuin A, Minderaa RB, Hoekstra PJ. Role of perinatal adversities on tic severity and symptoms of attention deficit/hyperactivity disorder in children and adolescents with a tic disorder. J Dev Behav Pediatr 2010; 31: 100-6.

21 Boyd A, Golding J, Macleod J, Lawlor DA, Fraser A, Henderson J, et al. Cohort Profile: the 'children of the 90s' - the index offspring of the Avon Longitudinal Study of Parents and Children. Int J Epidemiol 2013; 42: 111-27.

22 Fraser A, Macdonald-Wallis C, Tilling K, Boyd A, Golding J, Davey Smith G, et al. Cohort Profile: the Avon Longitudinal Study of Parents and Children: ALSPAC mothers cohort. Int J Epidemiol 2013; 42: 97-110.

23 Lawlor DA, Fraser A, Lindsay RS, Ness A, Dabelea D, Catalano P, et al. Association of existing diabetes, gestational diabetes and glycosuria in pregnancy with macrosomia and offspring body mass index, waist and fat mass in later childhood: findings from a prospective pregnancy cohort. Diabetologia 2010; 53: 89-97.

24 Lawlor DA, Macdonald-Wallis C, Fraser A, Nelson SM, Hingorani A, Davey Smith G, et al. Cardiovascular biomarkers and vascular function during childhood in the offspring of mothers with hypertensive disorders of pregnancy: findings from the Avon Longitudinal Study of Parents and Children. Eur Heart J 2012; 33: 335-45.

25 Brown MA, Lindheimer MD, de Swiet M, Van Assche A, Moutquin JM. The classification and diagnosis of the hypertensive disorders of pregnancy: statement from the International Society for the Study of Hypertension in Pregnancy (ISSHP). Hypertens Pregnancy 2001; 20: IX-XIV.

26 Rasmussen KM, Yaktine AL (eds) Weight Gain During Pregnancy: Reexamining the Guidelines. National Acadamies Press, 2009.

27 Miller LL, Scharf JM, Mathews CA, Ben-Shlomo Y. Tourette Syndrome and chronic tic disorder are associated with lower socio-economic status: findings from the ALSPAC cohort. Dev Med Child Neurol, in press.

28 Bobo JK, Husten C. Sociocultural influences on smoking and drinking. Alcohol Res Health 2000; 24: 225-32.

29 Kramer MS. Determinants of low birth weight: methodological assessment and meta-analysis. Bull World Health Organ 1987; 65: 663-737.

30 Riley EP, Infante MA, Warren KR. Fetal alcohol spectrum disorders: an overview. Neuropsychol Rev 2011; 21: 73-80.

31 Thapar A, Cooper M, Eyre O, Langley K. What have we learnt about the causes of ADHD? J Child Psychol Psychiatry 2013; 54: 3-16.

32 Langridge AT, Glasson EJ, Nassar N, Jacoby P, Pennell C, Hagan R, et al. Maternal conditions and perinatal characteristics associated with autism spectrum disorder and intellectual disability. PLOS One 2013; 8: e50963.

33 Millan MJ. An epigenetic framework for neurodevelopmental disorders: from pathogenesis to potential therapy. Neuropharmacology 2013; 68: 2-82.

34 Fraser A, Tilling K, Macdonald-Wallis C, Hughes R, Sattar N, Nelson SM, et al. Associations of gestational weight gain with maternal body mass index, waist circumference, and blood pressure measured $16 \mathrm{y}$ after pregnancy: the Avon Longitudinal Study of Parents and Children (ALSPAC). Am J Clin Nutr 2011; 93: 1285-92.
OPEN 